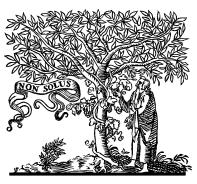

ELSEVIER MASSON
Disponible en ligne sur

ScienceDirect

www.sciencedirect.com
Elsevier Masson France

EM|consulte

www.em-consulte.com
NUTRITION CLINIQUE et MÉTABOLISME

Nutrition clinique et métabolisme $\mathrm{xxx}$ (2014) xxx-xxx

Développement professionnel continu

\title{
Comment gérer la nutrition artificielle chez un patient diabétique?
}

\author{
Management of artificial nutrition in diabetic patients \\ Nicolas Paquot $^{\mathrm{a}, *}$, Jenny DeFlines ${ }^{\mathrm{a}}$, Jean-Charles Preiser ${ }^{\mathrm{b}}$ \\ ${ }^{a}$ Service de diabétologie, nutrition et maladies métaboliques, CHU Sart-Tilman, 4000 Liège 1, Belgique \\ bépartement de soins intensifs, hôpital Erasme, 808, route de Lennik, 1070 Bruxelles, Belgique
}

\begin{abstract}
Résumé
Le développement d'une hyperglycémie chez des patients qui bénéficient d'une nutrition entérale ou parentérale représente un problème important en raison de sa haute prévalence et de ses conséquences en termes de morbi-mortalité. Cependant, la prise en charge du diabète ou d'une hyperglycémie de stress lors d'une nutrition artificielle constitue un domaine mal connu, en particulier chez le patient non agressé. Les indications de la nutrition artificielle et les voies d'accès ne diffèrent pas chez les patients diabétiques ou avec hyperglycémie de stress par rapport à un patient non diabétique. L'utilisation d'une formulation entérale spécifique au patient diabétique ne se justifie pas actuellement. Les objectifs glycémiques doivent être individualisés. Une glycémie préprandiale entre 100 et $140 \mathrm{mg} / \mathrm{dL}$ (5,5 et 7,8 mmol/L) et postprandiale entre 140 et $180 \mathrm{mg} / \mathrm{dL}$ (7,8 et $10 \mathrm{mmol} / \mathrm{L}$ ) apparaissent comme des objectifs raisonnables. Un contrôle régulier de la glycémie capillaire est indispensable. L'insuline constitue le traitement de choix pour traiter l'hyperglycémie et on recommande d'adapter la durée d'action théorique de l'insuline à la préparation nutritive. La prise en charge de ces patients nécessite le support d'une équipe multidisciplinaire expérimentée.
\end{abstract}

(c) 2014 Publié par Elsevier Masson SAS.

Mots clés : Diabète ; Hyperglycémie de stress ; Nutrition entérale ; Nutrition parentérale ; Patient non agressé

\section{Abstract}

Hyperglycaemia in patients receiving enteral or parenteral nutrition is a major problem due to its high prevalence and possible consequences in terms of morbidity and mortality. However, the management of diabetes/stress hyperglycaemia during artificial nutrition remains largely unknown, especially in non-critically ill patients. The indications and access routes for artificial nutrition are not different in patients with diabetes/stress diabetes than in non-diabetics. We do not recommend using enteral formulas designed for patients with diabetes. The glycaemic objective must be individualized. We recommend a preprandial blood glucose levels between 100 and $140 \mathrm{mg} / \mathrm{dL}(5.5$ and $7.8 \mathrm{mmol} / \mathrm{L})$ and postprandial levels between 140 and $180 \mathrm{mg} / \mathrm{dL}(7.8$ and $10 \mathrm{mmol} / \mathrm{L})$. A frequent monitoring of capillary glycaemias is mandatory. The best drug treatment for treating hyperglycaemia/diabetes is insulin and we recommend to adapt the theoretical insulin action to the nutrition infusion regimen. The management of these patients needs the help of a multidisciplinary experimented staff.

(C) 2014 Published by Elsevier Masson SAS.

Keywords: Diabetes; Stress hyperglycaemia; Enteral nutrition; Parenteral nutrition; Non-critically ill patients

\section{Introduction}

La nutrition artificielle chez des patients diabétiques ou qui présentent une hyperglycémie de stress constitue un sujet

\footnotetext{
* Auteur correspondant.

Adresse e-mail : nicolas.paquot@ulg.ac.be (N. Paquot).
}

important en nutrition clinique pour différentes raisons. Tout d'abord, la prévalence de l'hyperglycémie chez des patients hospitalisés qui bénéficient d'un support nutritionnel est extrêmement élevée et atteint, selon un relevé récent effectué dans 126 hôpitaux aux États-Unis (glycémie supérieure à $180 \mathrm{mg} / \mathrm{dL}$, $10 \mathrm{mmol} / \mathrm{L}$ ), $46 \%$ dans les unités de soins intensifs et $32 \%$ en dehors de ces unités [1]. Ensuite, l'hyperglycémie est associée à un pronostic péjoratif, tant chez les patients diabétiques que non 
diabétiques [2]. Plusieurs études ont été menées aux soins intensifs afin de déterminer si un contrôle précis et à des valeurs basses de la glycémie influençaient le pronostic des patients, mais les résultats continuent à faire l'objet de controverses [3-5]. Il semble que les effets délétères associés à l'hyperglycémie soient atténués chez le patient diabétique [6,7]. Enfin, le domaine de la nutrition artificielle chez le patient diabétique ou avec hyperglycémie de stress reste en grande partie mal connu et nécessiterait la réalisation d'études complémentaires, en particulier chez le patient en dehors des soins intensifs.

Récemment, différentes sociétés scientifiques ont proposé des recommandations pour la prise en charge de l'hyperglycémie au cours d'une nutrition artificielle chez des patients diabétiques ou non [8-13]. Il est à noter d'emblée que le niveau de preuves de ces recommandations est dans l'ensemble assez faible, témoignant du manque de données objectives face à cette situation clinique pourtant fréquente. De plus, la plupart des travaux ne font pas la distinction entre des patients diabétiques et ceux avec une hyperglycémie de stress, de même qu'entre patients agressés hospitalisés aux soins intensifs et patients non agressés séjournant en dehors de ces unités. Dans cette rubrique, nous nous proposons de synthétiser les recommandations les plus récentes concernant la gestion de la nutrition artificielle (entérale et parentérale) chez le patient diabétique ou avec hyperglycémie de stress, en se focalisant principalement sur le patient diabétique non agressé, hospitalisé en dehors du service des soins intensifs.

\section{Prévalence du diabète et de l'hyperglycémie chez des patients hospitalisés recevant une alimentation artificielle et conséquences}

Le nombre de diabétiques dans le monde est estimé actuellement à près de 250 millions et ce chiffre pourrait être de 380 millions d'ici dix ans environ. Le vieillissement de la population ainsi que le mode de vie actuelle (sédentarité, modifications de l'apport alimentaire) et des facteurs génétiques expliquent cette évolution quasi épidémique $[14,15]$. On estime que 15 à $25 \%$ des patients admis à l'hôpital sont diabétiques. La prévalence du diabète est encore plus importante chez les sujets âgés et qui résident en institution où près d'un tiers des patients âgés de $65-75$ ans présentent un diabète, se chiffre atteignant $40 \%$ chez les plus de 80 ans [16].

Chez des patients hospitalisés dans un contexte de maladie aiguë et dont le diabète n'est pas connu, une hyperglycémie de stress est définie par une glycémie à jeun $\geq 126 \mathrm{mg} / \mathrm{dL}$ $(7 \mathrm{mmol} / \mathrm{L})$ ou $\geq 200 \mathrm{mg} / \mathrm{dL}(11,1 \mathrm{mmol} / \mathrm{L})$ à n'importe quel moment avec une valeur d'hémoglobine glyquée $\left(\mathrm{HBA}_{1 \mathrm{C}}\right)$ inférieure à $5,7 \%$. Par définition, cette augmentation de la glycémie est transitoire et se normalisera lorsque le phénomène aigu disparaîtra. Certains patients demeureront néanmoins diabétiques. Comme le propose Olveira et al. [11], on pourrait identifier dès leur admission les sujets à risque de développer un diabète parmi ceux qui présentent une hyperglycémie avec une valeur d' $\mathrm{HBA}_{1 \mathrm{C}}$ entre $5,7 \%$ et $6,5 \%$, ce qui indique une anomalie dans le métabolisme du glucose préalable à l'admission. Une mesure de la valeur l'HBA ${ }_{1 C}$ serait dès lors souhaitable chez tous les patients hospitalisés avec une hyperglycémie ( $\geq 126 \mathrm{mg} / \mathrm{dL}$, $7 \mathrm{mmol} / \mathrm{L}$ ), afin de pouvoir préciser le risque de diabète ultérieur et de déterminer le mieux possible le traitement adéquat.

La relation entre l'hyperglycémie chez les patients hospitalisés (diabétiques ou non) et le risque accru de complications et de mortalité est bien établie (infections, complications cardiaques, sepsis, insuffisance rénale aiguë, décès) [17-20]. Bien que la plupart des études randomisées visant à investiguer l'impact du contrôle de la glycémie sur l'évolution clinique aient été conduites chez des patients hospitalisés aux soins intensifs, de nombreuses données observationnelles démontrent l'importance du traitement de l'hyperglycémie chez des patients admis dans les services généraux d'un hôpital [18-21]. Néanmoins, l'objectif glycémique à atteindre fait encore l'objet de nombreuses polémiques, en particulier chez le patient hospitalisé en soins intensifs. Les recommandations les plus récentes sont au moins concordantes sur un point: le maintien de la glycémie en dessous de $180 \mathrm{mg} / \mathrm{dL}$ (10 mmol/L), même si un seuil universel ne peut pas être défini [8,22]. Concernant le patient non agressé, un consensus actuel se dégage pour proposer de maintenir la glycémie préprandiale inférieure à $140 \mathrm{mg} / \mathrm{dL}$ $(7,8 \mathrm{mmol} / \mathrm{L})$ et une valeur inférieure à $180 \mathrm{mg} / \mathrm{dL}(10 \mathrm{mmol} / \mathrm{L})$ à tout moment. Ces objectifs peuvent bien entendu varier en fonction des situations cliniques rencontrées [11,12,23].

Chez le patient diabétique non agressé qui bénéficie d'une nutrition entérale (NE) ou parentérale (NP), l'objectif glycémique n'a pas fait l'objet d'études spécifiques et est donc laissé à l'appréciation du clinicien en fonction de la situation particulière de chaque patient [24]. L'objectif glycémique ne doit pas être trop strict, afin d'éviter la survenue d'hypoglycémies. Toutefois, comme mentionné ci-dessus, on observe une corrélation forte entre l'hyperglycémie induite par la nutrition parentérale totale (NPT) et une évolution clinique péjorative. De plus, une hyperglycémie supérieure à $150 \mathrm{mg} / \mathrm{dL}(8,3 \mathrm{mmol} / \mathrm{L})$ avant et au cours des 24 premières heures de l'initiation de la NPT est prédictive à la fois de la survenue de complications à l'hôpital et de mortalité [2]. Ces données suggèrent qu'une intervention précoce pour prévenir et corriger l'hyperglycémie pourrait améliorer l'évolution clinique des patients bénéficiant d'une nutrition artificielle [12]. En situation chronique, plusieurs sociétés scientifiques recommandent de maintenir la glycémie entre 140-180 mg/dL (7,8-10 mmol/L), même si aucune étude clinique contrôlée randomisée n'a été jusqu'à présent conduite en dehors des soins intensifs [11,13,23].

On estime qu'environ 5 à $8 \%$ des patients hospitalisés bénéficient d'un support nutritionnel artificiel entéral pour traiter ou prévenir la dénutrition, sous forme de suppléments ou pour couvrir la totalité des apports nutritionnels [11]. Environ 2-3\% de patients supplémentaires bénéficient d'une NPT pour les mêmes raisons.

L'hyperglycémie est une complication courante chez les patients hospitalisés qui bénéficient d'une NE, entre 34 et $50 \%$ des patients, variant notamment en fonction du type de population étudiée [25-27]. En ce qui concerne la NPT, on a rapporté entre 16 et $30 \%$ de sujets préalablement diabétiques chez les individus des unités de soins intensifs randomisés pour participer aux études d'intervention comparant le traitement par 
insuline conventionnel et intensif [11]. En revanche, la prévalence d'une l'hyperglycémie ou d'un diabète préalable chez des patients bénéficiant d'une NPT en dehors des unités de soins intensifs n'est pas connue. Pleva et al. [28] rapportent des épisodes hyperglycémiques à plus de $150 \mathrm{mg} / \mathrm{dL}(8,3 \mathrm{mmol} / \mathrm{L})$ chez plus de $90 \%$ de patients bénéficiant d'une NPT. D'autres travaux indiquent une prévalence de l'hyperglycémie supérieure à $200 \mathrm{mg} / \mathrm{dL}(11,1 \mathrm{mmol} / \mathrm{L})$ chez 12 à $27 \%$ des patients qui reçoivent une NPT [29,30], tandis qu'une étude espagnole récente (non publiée in extenso) rapporte que $51 \%$ des patients non agressés qui allaient bénéficier d'une NPT présentaient des anomalies du métabolisme du glucose (basées sur les valeurs de glycémies et d' hémoglobines glyquées) avant de débuter la NPT [31]. De plus, des études à la fois rétrospectives et prospectives démontrent que la mise en route d'une NE et d'une NP est un facteur de risque indépendant de l'apparition ou de l'aggravation d'une hyperglycémie, indépendamment d'une histoire préalable de diabète et que ceci est associé à un accroissement du risque de morbi-mortalité chez ces patients [12,20]. Concernant les mécanismes impliqués, l'hyperglycémie per se, outre le fait qu'elle révèle la gravité de la maladie sous-jacente, entraîne des conséquences défavorables majeures qui affectent le pronostic du patient hospitalisé, incluant l'augmentation de la mortalité, le taux d'infection et la durée de séjour à l'hôpital.

Il apparaît donc que l'hyperglycémie chez les patients qui bénéficient d'une nutrition artificielle, et tout spécialement chez les patients qui présentent un diabète avant la mise en place du support nutritionnel, constitue un problème important suite à sa prévalence élevée et les conséquences possibles en termes de morbi-mortalité. Il importe dès lors que ces patients soient pris en charge par des équipes nutritionnelles expérimentées possédant des compétences spécifiques en nutrition clinique et en diabétologie afin d'utiliser au mieux les traitements hypoglycémiants susceptibles de favoriser l'efficacité optimale de la nutrition artificielle utilisée. Les indications et les voies d'accès de la nutrition artificielle ne diffèrent pas chez les patients diabétiques (ou développant une hyperglycémie de stress) par rapport aux individus non diabétiques (sauf dans le cas particulier de la gastroparésie diabétique nécessitant une administration des nutriments au niveau post-pylorique).

\section{Traitement de l'hyperglycémie chez les patients non agressés diabétiques/avec hyperglycémie de stress et nutrition entérale}

L'obtention des objectifs glycémiques chez un patient diabétique recevant une NE constitue le plus souvent un vrai défi. Différentes circonstances, telles qu'un déplacement de la sonde, un arrêt imprévu ou non de l'administration (intolérance, examens médicaux, administration de médicaments, soins, kiné...) ou encore une administration cyclique de la NE avec un apport oral variable en fonction de l'appétit, font que la prescription d'un schéma d'insuline chez ces patients demeure le plus souvent problématique et va nécessiter l'assistance d'une équipe expérimentée.

Le meilleur schéma d'administration d'insuline chez des patients diabétiques qui bénéficient d'une NE ne peut être déterminé actuellement compte tenu du peu d'études disponibles. Il n'existe en réalité qu'une seule étude randomisée qui compare deux stratégies différentes d'administration d'insuline chez des patients hyperglycémiques non agressés - insuline rapide injectée selon un algorithme préétabli en fonction du monitoring glycémique (avec si nécessaire insuline NPH en supplément) comparée à de l'insuline rapide en combinaison avec de l'insuline glargine [27]. Les deux stratégies démontrent globalement une efficacité comparable. D'autres études rétrospectives impliquant un petit nombre de sujets ont été publiées, mais ne permettent pas de tirer des conclusions générales [11]. Les recommandations actuelles, reprises par différentes société scientifiques, sont donc basées sur des avis d' experts [11-13]. En nous inspirant de ces recommandations, nous pouvons proposer la synthèse ci-dessous.

Il est important tout d'abord de rappeler que les choix doivent être fait au cas par cas, notamment en fonction du mode d'administration de la NE (continue, cyclique ou en bolus). Afin d'éviter les risques d'hypoglycémie, on conseille de faire coïncider la durée d'action de l'insuline avec la durée de passage du mélange nutritif. Dans cette même optique, certains auteurs suggèrent que l'administration de quantités modérées et fréquentes d'insuline à action intermédiaire (type insuline NPH ou biphasique) seraient bénéfiques au niveau de la réduction du risque d'hypoglycémie, en combinaison si nécessaire avec de l'insuline à action rapide (analogue ou non). L'utilisation d'analogues de l'insuline à action prolongée (analogues lents) serait réservée aux patients avec des besoins plus stables ou chez les patients qui reçoivent la NE en bolus et chez lesquels l'insuline glargine est combinée à des analogues à action rapide [11,12].

Les Fig. 1-3 résument le traitement par insuline qui peut être proposé pour contrôler l'hyperglycémie lors d'une NE chez un patient diabétique. Lorsque la NE est administrée en bolus et si le sujet diabétique était traité préalablement avec des anti-diabétiques oraux, certains auteurs recommandent de commencer l'insuline sous-cutanée à une dose de 0,3-0,5 UI par $\mathrm{kg}$ de poids ajusté et par jour (en tenant compte également d'autres paramètres, tels que des pathologies intercurrentes ou d'autres traitements comme l'administration de corticoïdes) [11]. Si le patient était déjà traité par de l'insuline, la dose sera personnalisée en se basant sur les doses d'insuline préalables et la situation clinique. La surveillance glycémique doit être très régulière à l'instauration de la NE (au minimum un contrôle/3 heures). Le rapport insuline basale/insuline prandiale est à déterminer individuellement, mais on conseille, au début au moins, de ne pas administrer plus de $40 \%$ de la dose d'insuline totale sous forme basale, afin de réduire le risque d'hypoglycémie si la nutrition devait être interrompue brutalement [11]. On recommande de plutôt privilégier au départ l'insuline NPH ou détemir, l'insuline glargine pouvant être utilisée lorsque le patient est stable ou encore lorsque la quantité souhaitée de NE est atteinte. Concernant l'insuline prandiale, les analogues rapides seront privilégiés et administrés juste au démarrage de la poche de NE. Lorsque la NE est administrée de manière continue, une insuline basale est recommandée (glargine une fois par jour ou NPH, détémir ou biphasique deux ou trois fois par jour) associée à une insuline rapide ordinaire trois 


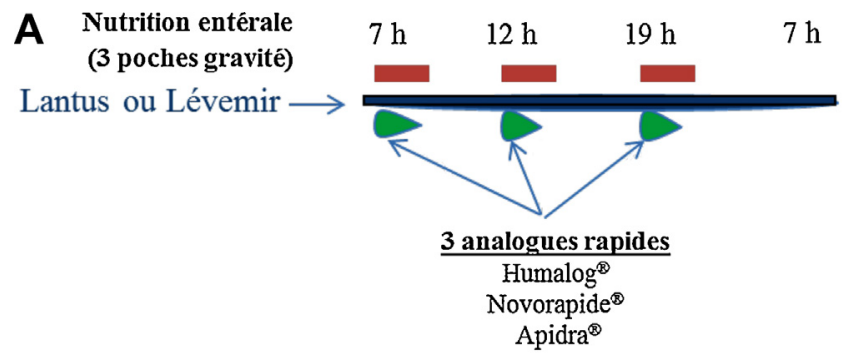

B

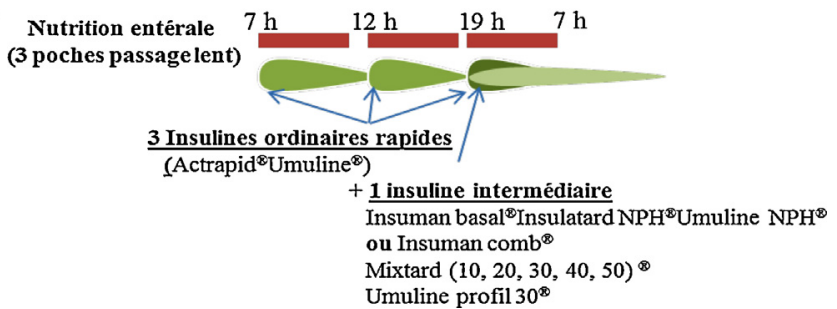

Fig. 1. Schémas d'insulinothérapie pour une nutrition entérale en 3 poches par jour. A. Passage de la nutrition entérale rapide en gravité $(<2 \mathrm{~h})$. Schéma proposé «basal bolus » : Lantus ${ }^{\circledR}$ ou Levemir ${ }^{\circledR}$ en basal, une fois par jour +3 injections d'analogues rapides avant chaque poche. Alternative : 3 injections d'Humalog ${ }^{\circledR}$ mix 30-50. B. Passage de la nutrition entérale sur plus de 4 heures. Deux schémas proposés: 3 injections d'insuline rapide ordinaire et une injection d'insuline intermédiaire Neutral Protamin Hagedorn (NPH) ; 3 injections d'un mélange mixte (mix 30-50). Le choix de la proportion d'analogue 30 ou 50 est fonction de la glycémie faite $1 \mathrm{~h} 30$ après le branchement de la poche.

D'après D. Quilliot et al. [32].

fois par jour dont la dose pourra être ajustée en fonction des contrôles glycémiques réguliers. Enfin, chez les patients bénéficiant d'une administration de NE cyclique (par exemple la nuit), on recommande une injection d'insuline à action intermédiaire (NPH, détémir ou biphasique) administrée 30 à 60 minutes avant le début de la perfusion (avec d'éventuels ajustements avec de l'insuline rapide ordinaire ou analogue au début de la poche).

Les préparations «standard» de NE contiennent habituellement une proportion élevée de glucides (environ $50 \%$ ) et un apport assez modéré en lipides (environ $30 \%$ ), ce qui favoriserait une réponse glycémique et insulinémique élevée chez le sujet diabétique par comparaison à ce qui est observé après un repas mixte. Dès lors, différentes préparations spécifiques destinées

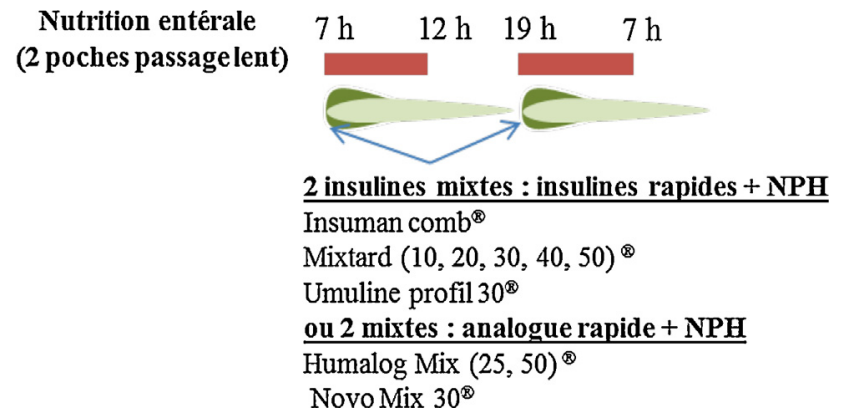

Fig. 2. Schémas d'insulinothérapie pour une nutrition entérale en 2 poches par jour. Schéma proposé : 2 injections d'une insuline mixte (association d'une insuline rapide ou d'un analogue et d'une insuline NPH). Le choix de la proportion d'analogue ou de rapide 30 ou 50 est fonction de la glycémie réalisée $1 \mathrm{~h} 30$ après le branchement de la poche.

D’après D. Quilliot et al. [32].

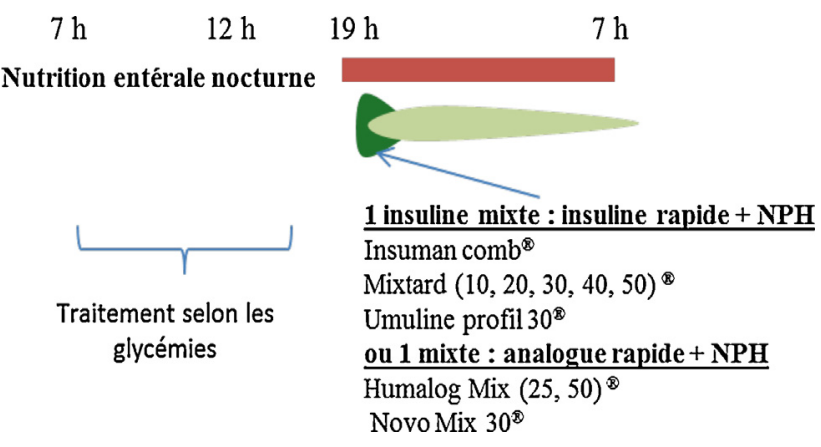

Fig. 3. Schémas d'insulinothérapie pour une nutrition entérale cyclique nocturne. Schéma proposé : une injection d'un mélange d'insuline mixte (association d'une insuline rapide ou d'un analogue et d'une insuline NPH). Le choix de la proportion d'analogue ou de rapide 30 ou 50 est fonction de la glycémie réalisée $1 \mathrm{~h} 30$ après le branchement de la poche. En nutrition entérale continue, au long cours : possibilité d'utiliser une insuline de type Lantus ${ }^{\circledR}$ (longue durée d'action).

D'après D. Quilliot et al. [32].

aux patients diabétiques ont été mises sur le marché au cours de ces dernières années. Leur principe est de fournir des glucides à index glycémique bas avec une quantité plus importante de fibres solubles. De plus, ces préparations contiennent un pourcentage plus élevé de lipides (principalement sous la forme d'acides gras monoinsaturés). L'avantage principal de ce type de préparations serait de réduire l'hyperglycémie postprandiale. Et de fait, des études cliniques menées à l'hôpital ont montré une diminution des glycémies et une réduction des besoins en insuline, mais sans modifier les paramètres déterminants, tels que la durée du séjour hospitalier, la survenue des infections et la mortalité [11]. Sur des périodes plus longues (jusqu'à trois mois), ces préparations spécifiques pour patients diabétiques amélioreraient le contrôle métabolique (en particulier la valeur d'HBA $\mathrm{HC}_{1 \mathrm{C}}$ ), mais sans modifier la morbidité [33]. Cependant, même si une métaanalyse est en faveur de l'usage de ces préparations entérales spécifiques pour les sujets diabétiques [34], il manque actuellement d'études randomisées, de préférence menées en double insu et incluant plus de patients sur une durée plus longue, qui permettraient d'obtenir des résultats probants en termes de morbi-mortalité. Dès lors, dans ces dernières recommandations, l'ASPEN conclut qu'il n'existe pas de données suffisantes pour recommander l'usage de telles préparations chez les patients adultes hospitalisés avec hyperglycémie [13].

\section{Traitement de l'hyperglycémie chez le patient diabétique non agressé recevant une nutrition parentérale totale (NPT)}

En dehors des unités de soins intensifs, la gestion de l'hyperglycémie au cours d'une NPT n'a pas fait l'objet d'études randomisées, mais deux articles récents y ont été consacrés dans cette revue et le lecteur peut s'y référer [24,32].

$\mathrm{Au}$ niveau de la composition en macronutriments de la poche de NPT, il n'existe pas d'étude qui permette d'établir la proportion idéale. Tant qu'à présent, on recommande un apport de calories non protéiques entre $60 \%$ de glucides et $40 \%$ de lipides. Le type de macro- ou de micronutriments à 
$7 \mathrm{~h}$

$12 \mathrm{~h}$

$19 \mathrm{~h}$

Nutrition parentérale nocturne
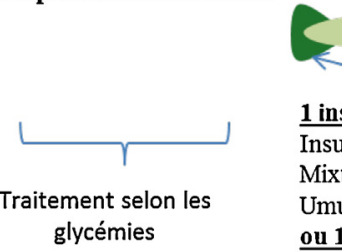

1 insuline mixte : insuline rapide + NPH Insuman comb ${ }^{\otimes}$

Mixtard $(10,20,30,40,50)$

Umuline profil $30^{\circledR}$

ou 1 mixte : analogue rapide + NPH

Humalog Mix $(25,50)^{2}$

Novo Mix $30^{3}$

Fig. 4. Schémas d'insulinothérapie en nutrition parentérale cyclique nocturne. Une injection d'un mélange d'insuline mixte (association d'une insuline rapide ou d'un analogue et d'une insuline NPH). Le choix de la proportion d'analogue ou de rapide 30 ou 50 est fonction de la glycémie réalisée $1 \mathrm{~h} 30$ après le branchement de la poche.

D'après D. Quilliot et al. [32].

administrer chez les sujets diabétiques en NPT n'a pas fait non plus l'objet d'études spécifiques. Bien que l'utilisation de mélanges particuliers d'acides aminés (par exemple riches en glutamine) ou de lipides spécifiques (basé sur l'acide oléique ou enrichis en acides gras oméga-3) pourrait se montrer bénéfique dans la prise en charge de l'hyperglycémie, il n'existe pas actuellement de données suffisantes dans la littérature pour en recommander leur utilisation.

La Fig. 4 résume le traitement par insuline qui peut être proposé pour contrôler l'hyperglycémie lors d'une NPT chez un patient diabétique. En situation chronique et stable, l'administration d'insuline rapide ordinaire directement dans la poche de NPT est une pratique courante et fait l'objet d'un large consensus, bien que cette pratique n'ait fait l'objet d'aucune évaluation précise et que la biodisponibilité exacte de l'insuline associée à la poche de NPT reste mal connue $[12,24,28]$. Cette technique nécessite néanmoins des précautions importantes: elle doit être initiée en hospitalisation et les conditions d'asepsie doivent être strictement contrôlées du fait des risques infectieux (Encadré 1). L'avantage indéniable de ce type d'administration est de limiter le risque d'hypoglycémie en cas d'arrêt inopiné de la NPT [32]. Une pratique clinique assez courante est d'administrer l'insuline dans la poche et de corriger l'hyperglycémie éventuelle - une autosurveillance glycémique régulière est indispensable - avec des injections d'insuline rapide ordinaire sous-cutanée toutes les 6-8 heures $[11,12]$. Si l'objectif glycémique n'est pas atteint, l'équivalent des deux tiers de la dose de l'insuline sous-cutanée injectée en correction le jour précédent sera ajouté dans la perfusion quotidienne [11]. En cas de diabète connu non-traité par insuline préalablement, on peut administrer 1 à 2 UI d'insuline pour $10 \mathrm{~g}$ de glucose contenu dans la poche NPT. Si le diabète était déjà traité par insuline, on commence par 2 UI pour $10 \mathrm{~g}$ de glucose ou 0,5 UI d'insuline par $\mathrm{kg}$ de poids. Comme alternative, on peut débuter en administrant dans la poche de NPT la moitié ou les deux tiers des besoins antérieurs du patient. Chez les patients diabétiques de type 1, en plus de l'insuline administrée dans la poche et du complément sous-cutané, on recommande d'injecter entre 0,05 et $0,1 \mathrm{UI} / \mathrm{kg}$ d'insuline basale sous-cutanée (glargine ou détémir) afin de prévenir une décompensation acido-cétosique en cas d'arrêt impromptu de la perfusion [11].

\section{Encadré 1: Insuline injectée dans la poche de nutrition parentérale : les précautions et modalités}

\section{- La nutrition parentérale doit être débutée et éva- luée en hospitalisation;}

- On doit s'assurer d'une bonne homogénéité du mélange : retournement de la poche à de multiples reprises (le volume injecté, inférieur à $1 \mathrm{~mL}$ doit être dilué dans un volume généralement supérieur à $1 \mathrm{~L}$ ) ;

- La composition de la poche doit être constante: la biodisponibilité de l'insuline peut varier de façon considérable, selon la composition en micronutriments et en acides aminés. Par exemple si les oligo-éléments sont ajoutés dans le mélange, il faut que cette pratique soit constante ;

- Il faut utiliser une insuline de type rapide (Umuline ${ }^{\circledR}$, Actrapid $\left.^{\circledR}\right)$ (la compatibilité avec les analogues n'a pas été testée) ;

- La stabilité de la biodisponibilité de I'insuline n'est pas garantie, il est donc recommandé de contrôler les glycémies, toutes les 2 heures, durant les premiers jours, de façon à adapter les doses d'insuline;

- La dose initiale est généralement comprise entre 0,5 à $1 \mathrm{UI} / 10 \mathrm{~g}$ de glucose perfusé ;

- Un rattrapage de $2 U$ est effectué en sous-cutané si la glycémie est supérieure aux objectifs fixés. On tiendra compte de ces rattrapages pour calculer la dose du lendemain. L'adaptation de la dose d'insuline se fera ensuite sur la glycémie au débranchement.

Ce n'est qu'en cas d'hyperglycémie importante, persistante et non contrôlée qu'une administration intraveineuse séparée d'insuline devra être envisagée.

Chez les patients diabétiques qui bénéficient d'une NP à domicile avec administration cyclique (souvent nocturne), on peut envisager d'administrer avant la début de la perfusion une insuline intermédiaire (NPH, détémir ou insuline biphasique) par voie sous-cutanée.

\section{Conclusions}

La prise en charge du diabète chez les sujets qui bénéficient d'une nutrition artificielle reste un domaine mal connu, en particulier chez le patient non agressé. Actuellement, les recommandations se basent essentiellement sur des avis d'experts. De nouvelles études prospectives et incluant un nombre suffisant de patients sont indispensables afin mieux définir les objectifs glycémiques, le type de préparation à utiliser ou encore le(s) schéma(s) d'insuline à préconiser, pas seulement en termes de 
contrôle métabolique, mais aussi pour leur(s) effet(s) au niveau de la morbi-mortalité chez ces patients.

\section{Déclaration d'intérêts}

Les auteurs déclarent ne pas avoir de conflits d'intérêts en relation avec cet article.

\section{Références}

[1] Cook CB, Kongable GL, Potter DJ, Abad VJ, Leija DE, Anderson M. Inpatient glucose control: a glycemic survey of 126 US hospitals. J Hosp Med 2009;4:E7-14.

[2] Pasquel FJ, Spiegelman R, McCauley M, Smiley D, Umpierrez D, Johnson $\mathrm{R}$, et al. Hyperglycemia during total parenteral nutrition: an important marker of poor outcome and mortality in hospitalized patient. Diabetes Care 2010;33:739-41,

[3] Dungan KM, Braithwaite SS, Preiser JC. Stress hyperglycaemia. Lancet 2009;373:1798-807.

[4] Marik PE, Preiser JC. Toward understanding tight glycemic control in the ICU: a systematic review and meta-analysis. Chest 2010;137:544-51.

[5] Lena D, Kalfon P, Preiser JC, Ichai C. Glycemic control in the intensive care unit and during the postoperative period. Anesthesiology 2011;114:438-44.

[6] Vincent JL, Preiser JC, Sprung CL, Moreno R, Sakr Y. Insulin-treated diabetes is not associated with increased mortality in critically ill patients. Crit Care 2010;14(1):R12, http://dx.doi.org/10.1186/cc8866.

[7] Krinsley JS, Egi M, Kiss A, Devendra AN, Schuetz P, Maurer PM, et al. Diabetic status and the relation of the three domains of glycemic control to mortality in critically ill patients: an international multicenter cohort study. Crit Care 2013;17(2):R37.

[8] Ichai C, Preiser JC, Société française d'anesthésie-réanimation, Société de réanimation de langue française, Experts group. International recommendations for glucose control in adult non diabetic critically ill patients. Crit Care 2010;14:R166, http://dx.doi.org/10.1186/cc9258.

[9] Qaseem A, Humphrey LL, Chou R, Snow V, Shekelle P. Use of intensive insulin therapy for the management of glycemic control in hospitalized patients: a clinical practice guideline from the American College of Physicians. Ann Intern Med 2011;154:260-7.

[10] Vaquerizo Alonso C, Grau Carmona T, Juan Díaz M. Guidelines for specialized nutritional and metabolic support in the critically ill patient. Update. Consensus SEMICYUC-SENPE: hyperglycemia and diabetes mellitus. Nutr Hosp 2011;26(Suppl. 2):37-40.

[11] Olveira G, García-Luna PP, Pereira LJ, Rebollo I, García-Almeida JM, Serrano P, et al. Recommendations of the GARIN group for managing noncritically ill patients with diabetes or stress hyperglycaemia and artificial nutrition. Nutr Hosp 2012;27:1837-49.

[12] Umpierrez GE, Hellman R, Korytkowski MT, Kosiborod M, Maynard GA, Montori VM, et al. Management of hyperglycemia in hospitalized patients in non-critical care setting: an endocrine society clinical practice guideline. J Clin Endocrinol Metab 2012;97:16-38.

[13] McMahon MM, Nystrom E, Braunschweig C, Miles J, Compher C. ASPEN clinical guidelines: nutrition support of adult patients with hyperglycemia. J Parenter Enteral Nutr 2013;37:23-36.

[14] Hu FB. Globalization of diabetes: the role of diet, lifestyle, and genes. Diabetes Care 2011;34:1249-57.

[15] Scheen AJ, Paquot N. Le diabète de type 2: voyage au cœur d'une maladie complexe. Rev Med Liege 2012;67(5-6):326-31.

[16] Morley JE. An overview of diabetes mellitus in older persons. Clin Geriatr Med 1999; 15:211-24.
[17] Capes SE, Hunt D, Malmberg K, Gerstein HC. Stress hyperglycaemia and increased risk of death after myocardial infarction in patients with and without diabetes: a systematic overview. Lancet 2000;355:773-8.

[18] Umpierrez GE, Isaacs SD, Bazargan N, You X, Thaler LM, Kitabchi AE. Hyperglycemia: an independent marker of in-hospital mortality in patients with undiagnosed diabetes. J Clin Endocrinol Metab 2002;87:978-82.

[19] Falciglia M, Freyberg RW, Almenoff PL, D’Alessio DA, Render ML. Hyperglycemia-related mortality in critically ill patients varies with admission diagnosis. Crit Care Med 2009;37:3001-9.

[20] Olveira G, Tapia MJ, Ocón J, Cabrejas-Gómez C, Ballesteros-Pomar MD, Vidal-Casariego A, et al. Parenteral nutrition-associated hyperglycemia in non-critically ill inpatients increases the risk of in-hospital mortality. Diabetes Care 2013;36:1061-6.

[21] McAlister FA, Majumdar SR, Blitz S, Rowe BH, Romney J, Marrie TJ. The relation between hyperglycemia and outcomes in 2471 patients admitted to the hospital with community-acquired pneumonia. Diabetes Care 2005;28:810-5

[22] Jacobi J, Bircher N, Krinsley J, Agus M, Braithwaite SS, Deutschman, et al. Guidelines for the use of an insulin infusion for the management of hyperglycemia in critically ill patients. Crit Care Med 2012;40: 3251-76.

[23] Moghissi ES, Korytkowski MT, DiNardo M, Einhorn D, Hellman R, Hirsch IB, et al. American Association of Clinical Endocrinologists and American Diabetes Association consensus statement on inpatient glycemic control. Diabetes Care 2009;32:1119-31

[24] DeFlines J, Paquot N, Preiser JC. Gestion de l'hyperglycémie au cours d'une nutrition parentérale. Nutr Clin Metab 2012;26:143-7.

[25] Pancorbo-Hidalgo PL, Garcia-Fernandez FP, Ramirez-Perez C Complications associated with enteral nutrition by nasogastric tube in an internal medicine unit. J Clin Nurs 2001;10:482-90.

[26] Arinzon Z, Shabat S, Shuval I, Peisakh A, Berner Y. Prevalence of diabetes mellitus in elderly patients received enteral nutrition long-term care service. Arch Gerontol Geriatr 2008;47:383-93.

[27] Korytkowski MT, Salata RJ, Koerbel GL, Selzer F, Karslioglu E, Idriss $\mathrm{AM}$, et al. Insulin therapy and glycemic control in hospitalized patients with diabetes during enteral nutrition therapy: a randomized controlled clinical trial. Diabetes Care 2009;32:594-6.

[28] Pleva M, Mirtallo JM, Steinberg SM. Hyperglycemic events in nonintensive care unit patients receiving parenteral nutrition. Nutr Clin Pract 2009;24:626-34.

[29] Valero MA, Alegre E, Gomis P, Moreno JM, Miguelez S, Leon-Sanz M. Clinical management of hyperglycaemic patients receiving total parenteral nutrition. Clin Nutr 1996;15:11-5.

[30] Marti-Bonmati E, Ortega-Garcia MP, Cervera-Casino P, Lacasa C, Llop JL, Villalobos JL, et al. Multicenter study on the prevalence of hyperglycemia among hospitalized patients with parenteral nutrition. Farm Hosp 2006:30:12-9.

[31] Tapia Guerrero MJ, Olveira G, Hyperglycaemia in Parenteral Nutrition Study Group. Hyperglycemia and diabetes during total parenteral nutrition in non-critical hospitalised patients in Spain. Clin Nutr 2011;6(Supp. 1): 120 .

[32] Quilliot D, Durain D, Malgras A. Principes de prise en charge de l'hyperglycémie au cours de la nutrition artificielle. Nutr Clin Metab 2012;26:40-4.

[33] Vaisman N, Lansink M, Rouws CH, van Laere KM, Segal R, Niv $\mathrm{E}$, et al. Tube feeding with a diabetes-specific feed for 12 weeks improves glycaemic control in type 2 diabetes patients. Clin Nutr 2009;28: 549-55.

[34] Elia M, Ceriello A, Laube H, Sinclair AJ, Engfer M, Stratton RJ. Enteral nutritional support and use of diabetes-specific formulas for patients with diabetes: a systematic review and meta-analysis. Diabetes Care $2005 ; 28: 2267-79$. 